\title{
StratosPheric OzOne, Middle UltraViolet Radiation and Phytoplankton Productivity
}

\author{
By Raymond C. Smith and Karen S. Baker
}

$\mathrm{M}$ ARINE LIFE IN THE UPPER layers of the sea may be endangered by increased ultraviolet (UV) radiation resulting from reduction in the thickness of the earth 's ozone layer. There is undisputed observational evidence that human activities, including the production of chlorofluorocarbons, are influencing the concentration of stratospheric ozone (Watson, 1988). A significant reduction in average global stratospheric ozone is predicted over the next century, despite international efforts to address the problem. Seasonal depletions of ozone in the Antarctic during austral spring have already reached $50 \%$. resulting in the widely discussed "ozone hole" (Brasseur. 1987). Reduced stratospheric ozone will result in increased biologically damaging ultraviolet radiation ( $U V$ - B, 280-320nm) reaching the surface of the earth. Since UV-B radiation can penetrate to ecologically significant depths in water (Jerlov, 1950; Lenoble, 1956: Smith and Baker. 1979: Gieskes et (al. 1989), any ozone reduction results in increased UV-B penetrating the surface layers of marine environments.

The atmospheric component ozone, $\mathrm{O}_{3}$, is a strong selective absorber of ultraviolet radiation (UVR. 200-400nm). The shortest UV wavelengths. UV-C $(200-280 \mathrm{~nm})$. while most damaging to organisms, are absorbed strongly by the atmosphere so that negligible amounts reach the earth's surface. Middle ultraviolet radiation. UV-B $(280-320 \mathrm{~nm})$, is extremely injurious to organisms and increases when atmospheric ozone is reduced. UV-A (320-400nm). a region of the solar spectrum that has been shown to play a role in both photodamage and photorepair. is essentially unaltered by ozone depletion. Similarly, photosynthetically available radiant energy (PAR, $400-700 \mathrm{~nm}$ ), the spectral region essential for both photoprotection and photosynthesis, is unaltered by ozone depletion. As a consequence. stratospheric ozone depletion leads to a dramatic increase in damaging wavelengths within the UV-B portion of the spectrum. while energy required to drive photorepair processes, either through UV-A photoreactiva-

Raymond C. Smith, Universily of California Marine Bio-Opties, CSL/Center for Remote Sensing and Envronmental Optics. University of California. at Santa Barbara. Santa Barbara. CA 93106: and Karen S. Baker. Lniversity of Califorma Marine BioOptics, Scripps Institution of Oceanography, A-018. University of Californiat. at San Dego. La Jolla. CA 92093. tion mechanisms or PAR-dependent photosynthetic processes, remains constant. It is likely that the degree of photodamage to an organism is a function of the shifting balance between hazardous UV-B exposure and the photoprotective capabilities of the organism provided by longer wavelengths.

Laboratory lindings show some algae as well as larval stages of fishes and invertebrates are extremely sensitive to UV-B radiation. The extrapolation of laboratory findings to prediction of possible ultraviolet radiation impacts on natural communities of marine organisms is replete with uncertainty. As a consequence, there is a pressing need for direct measures of ultraviolet radiation effects on natural aquatic communities in order to reliably assess the potential environmental impact. Recent relevant reviews include: NAS (1984). Caldwell et al. (1986), Worrest (1986), NOAA (1987), Voytek (1989) and Watson (1988).

General Effects of UV-B on Aquatic Organisms

A major body of literature exists on the detrimental effects of UV radiation on the physiology and biochemistry of many forms of life (Lrbach. 1969; Giese, 1976: Jagger, 1985). Death and mutagenesis are the most dramatic of the deleterious effects of UV radiation. In general, the shorter the wavelength, the greater and more itreversible the damage. Several pioneering investigations demonstrated the damaging effects of ultraviolet radiation on aquatic organisms. but they lacked precise and accurate determinations of UV fluence. The assessment of the ecological consequences of $U V$ radiation is complicated by the exacting dosimetry necessary and by the uncertainty with respect to the photoprocesses, and corresponding biological weighting function, responsible for damage and repair. In order to estimate the possible damage to aquatic organisms, it is necessary to determine the biologically weighted fluence rates at depth, which depend upon: the spectral UVR incident at the surface: the optical water type. which determines the spectral penetration of radiation to depth: a biological weighting function for UVR damage: and the mixing rate and depth distribution of organisms, which determines the time an organism spends at depth.

\section{Biological Weighting Function}

A fundamental law of photochemistry states that 

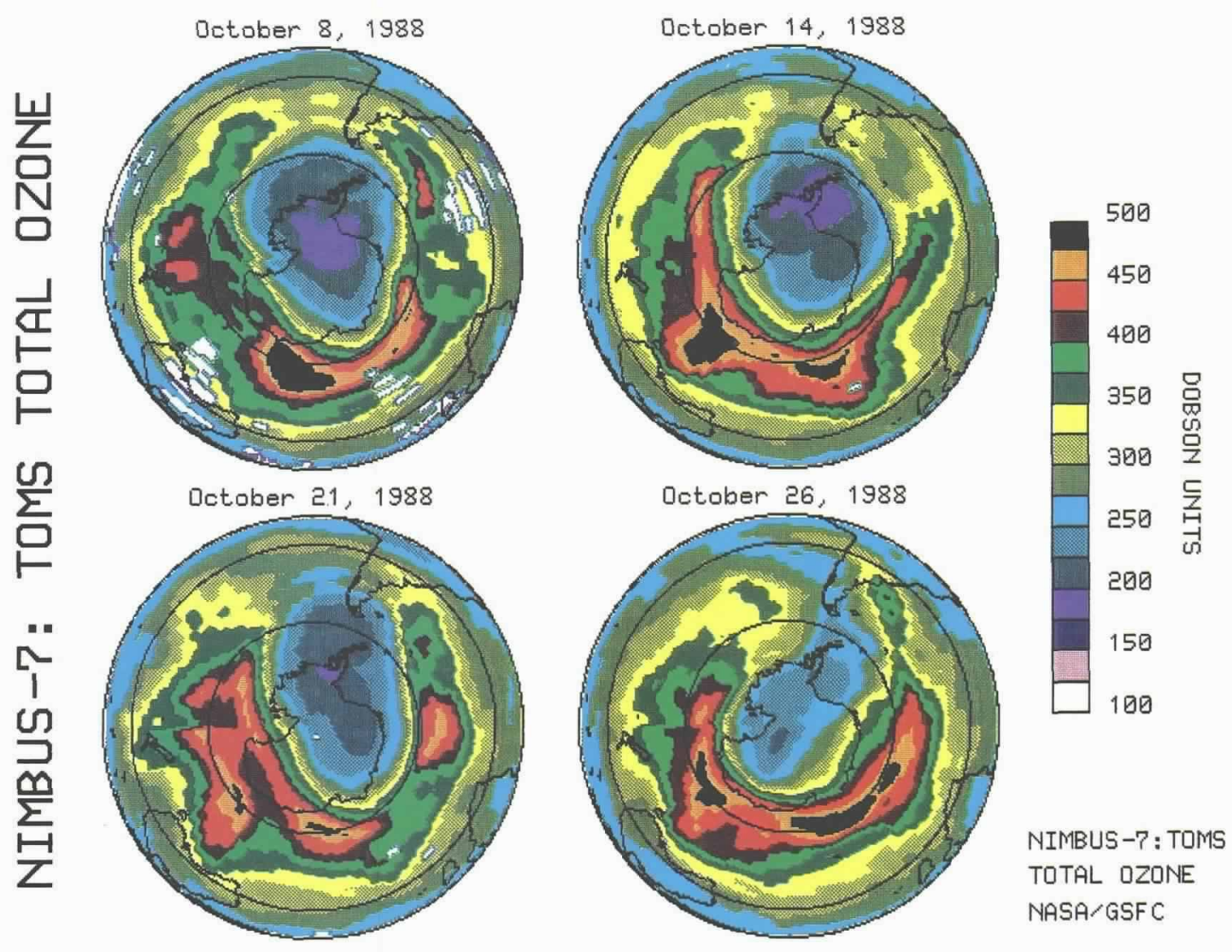

Fig. 3: Total Ozone Mapping Spectrometer (TOMS) satellite imagery of the Antarctic ozone hole on (a) October 8, 1988 (Julian day (jd) 282), (b) October 14,1988 (jd 288), (c) October 21,1988 (jd 295), (d) October 26, 1988 (jd 300). Ozone concentrations are in color-coded Dobson Units (DU). The images illustrate that the hole is elongated, not centered on the geographic pole, and variable as a function of time. As a consequence, there is a spatial region of enhanced $U V$-B that varies as a function of time. White patches indicate missing data.

for a reaction to occur a photon must be absorbed. To assess the impact of ozone reduction, it is important to know the spectral nature of the relative biological efficiency for the absorption of photons for the particular photoeffect under consideration. Determination of the biologically effective radiation is based upon a weighting function, or action spectrum, that takes account of the wavelength dependence of the biological photoprocess under consideration. Ideally, a biological weighting function should be determined for the organism studied, but it has been more practical to search for generalized weighting functions (Rundel, 1983; Caldwell et al., 1986). While there is a great variety of physiological and morphological phenomena attributable to UV-B radiation, the principal absorbing compounds for the diversity of plant and animal responses may have some similarity. For example, it has been observed that action spectra commonly conform to the absorption spectra of either proteins or nucleic acids. A relative biological efficiency for biological effects involving DNA, the molecule responsible for the transmission of genetic information, $\varepsilon_{\mathrm{DNA}}$, is heavily weighted in the UV-B region of the spectrum (Fig. 1, p. 6). In contrast, the relative biological efficiency for photoinhibition of chloroplasts, $\varepsilon_{\mathrm{pl}}$, shows significant absorption throughout the ultraviolet and visible spectrum (Fig. 1). Caldwell et al. (1986) have proposed a biological weighting function that lies between these two relatively extreme functions.

Also shown in Fig. 1 is a plot of solar radiation incident at the surface of the ocean for two stratospheric ozone concentrations given in Dobson Units (DU). Labeled curves are shown for a normal midlatitude concentration (320 DU) and for a reduced ozone concentration ( $240 \mathrm{DU})$. The additional radiation resulting from the diminished ozone concentration is confined to the UV-B region of the spectrum and makes an insignificant contribution to the total broadband energy as well as to the photosynthetically available radiant energy (400-700nm), PAR.

The biologically weighted fluence rate for a particular photoeffect is the product of the appropriate biological efficiency and the spectral irradiance 
$A_{\text {critical factor ... }}$

is an accurate estimate

of the biological

weighting function.

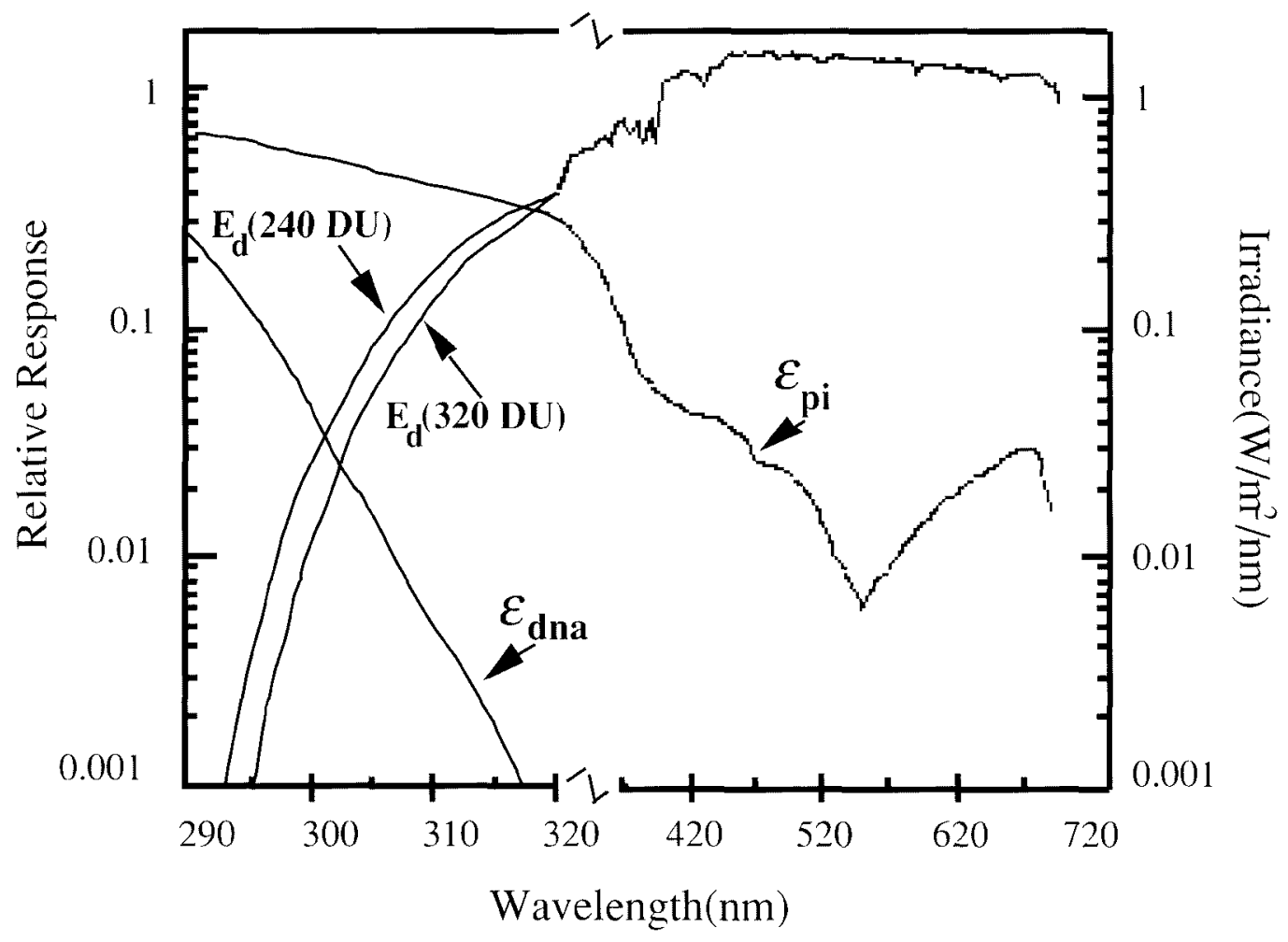

Fig. 1: Relative biological efficiencies. $\varepsilon_{f t}(\lambda)$ and $\varepsilon_{D N_{A}}(\lambda)$, and downward spectral irradiance. $E(\lambda)$. for two stratospheric ozone concentrations [240 DU, 320 DU] plotted against wavelength [nm]. Note that the wavelength scale is expanded below 320 mm in order to show greater detail in the UV'B region of the spectrum.

integrated over wavelength. The biological fluence rate for a particular photoeffect can increase significantly due to depleted ozone if the biological weighting function is weighted toward radiation in the UV-B region (e.g., Fig. I DNA) but would be relatively insignificant for weighting functions that have significant absorption outside the UV-B spectral region of ozone absorption. Thus, a critical factor in assessing the possible influence of increased UV-B on aquatic organisms is an accurate estimate of the biological weighting function.

Researchers conducting laboratory experiments for the assessment of UV-B damage frequently attempt to measure an effect (such as reduced growth rates, changes in pigment composition. specific damage, or death of an organism) versus biologically weighted fluence rate (NAS. 1984). Typically, up to a threshold fluence rate. there is no measurable damage to the organism. As the fluence rate is increased beyond this level. an increasing percentage of the organisms are affected. This data describes a "survival curve" which provides an estimate not only of the threshold level but also the level lethal to $50 \%$ of the organisms $L_{51 !}$. These levels can be compared with current or predicted future fluence rates to assess the possible influence of natural radiation on the organism.

\section{Extrapolation of Laboratory Findings}

There are several methodological problems associated with attempting to link laboratory and field observations to possible consequences of solar UV-B radiation change on natural populations. First, biological weighting functions have traditionally been determined by evaluating biological responsiveness to monochromatic (i.e., narrowband) radiation with the objective of identifying potential pigment targets and elucidating photobiological mechanisms. Caldwell et al. (1986) review evidence to show that weighting functions determined using polychromatic (i.e., broadband) radiation and intact organisms are of more ecological relevance. Broadband radiation more nearly approximates the total influence, both beneficial and detrimental. of all wavelengths present in natural sunlight. Second, a critical problem in discussing survival curves concerns the biological dose, which is the product of an irradiance rate and the length of time of the biological exposure. The dose does not always cause an equal effect for a short exposure to a high intensity source as a long exposure to a low intensity source even though the products are 
equal. This is analogous to the "reciprocity problem" familiar to photographers (Mees, 1954). Presumably lack of reciprocity is related to the time rates and spectral responses of repair mechanisms in relation to the time rates and spectral weighting functions of the damaging radiation. A third critical factor in the methodology of laboratory experiments is that radiation regimes in laboratory experiments cannot easily simulate ambient levels of solar radiation throughout the total spectrum. While experiments may enhance the UV-B spectral region, the visible portion of the spectrum may be as much as an order of magnitude lower than in nature, which limits the energy for optimum photoreactivation and photorepair. Fourth. there is evidence that the sum of effects of enhanced UV-B may he non-linear and can be antagonistic or synergistic with respect to other photoprocesses. These issues confound efforts to extrapolate results of laboratory experiments to natural communities. All evidence should be viewed in the context of these methodological problems.

\section{Phytoplankton}

There is convincing evidence that UV-B radiation, at levels currently incident at the surface of the ocean, has an influence on phytoplank ton productivity (Harris, 1978: Smith et al.. 1980): Worrest, 1986). Early ${ }^{14} \mathrm{C}$ work with fixed bottles composed of UVtransmitting and non-transmitting materials demonstrated that the ultraviolet component of solar radiation inhibited uptake rates, but did not allow for the quantitative estimation of the biological weighting function responsible for the observed results. Smith ct al. (1980) and Smith and Baker (1980.1982) found the $\varepsilon_{\mathrm{pl}}$ action spectra (Fig. 1) was consistent with their results. but they stressed that time-dependent processes, such as damage to DNA or the production of UV-B screening pigments, would not be observed in productivity incubations that are short compared with the generation time of the organisms under study. Short-term incubations may be inadequate for accurately assessing the long-term ecological consequences of increased UV-B on phytoplankton communities. These workers stated the limitations of a linear model as well as the result which estimates that a $25 \%$ depletion in stratospheric ozone at temperate latitudes would result in a $9 \%$ decrease in marine primary productivity integrated over the entire euphotic zone.

Calkins and Thordardottir (1980) made a comparison of solar UV effects at high and low latitudes in order to assess the lethal limit of organisms to levels of UV flux in their nomal habitats. The natural concentration of stratospheric ozone varies with latitude, being thinner in the tropics with corresponding greater atmospheric penetration of UV-B than at higher latitude and is highly dependent on solar angle as well. As a consequence, UV-B radiation at high latitudes is roughly half the maximum daily amount reaching temperate and tropical latitudes. Calkins and Thordardottir, comparing observations for aquatic ecosystems in Iceland $\left(63^{\circ} \mathrm{N}\right)$ and Kentucky $\left(36^{\circ} \mathrm{N}\right)$, inferred that solar UV is a significant ecological factor because there was little or no reserve capacity to withstand increased UV dosage in either habitat. They argue that while organisms may have photoadaptive capacity or the ability to avoid solar UV, any adaptation to solar UV reduces the resources available for other purposes. This suggests that current levels of UV-B are an already occurring natural stress for phytoplankton communities.

Worrest and co-workers (Worrest. 1986), using naturally derived microcosms, explored whether predicted increases in UV-B radiation might overwhelm natural protective mechanisms and produce changes that would have damaging consequences to the ecosystem. In agreement with others, they found that acute exposure to UV-B radiation significantly depressed the radiocarbon estimate of primary productivity and that various phytoplankton species showed a differential sensitivity to UV-B radiation. Their results suggest that long-term studies, and by inference natural communities, would show alteration of algal community structure as a result of exposure to enhanced levels of UV-B radiation. Further, a DNA-like biological weighting function was most consistent with their experiments.

Jokiel and York (1984) also focused on experiments that tested long-term cological effects. They used monoculture experiments with natural sunlight to determine the influence of current levels of UV-B and PAR on the growth of microalgae. Over a period of one to two weeks, little or no photoinhibition of growth rate in the absence of UV radiation was found. They concluded that when long-term growth inhibition was observed. it was almost entirely caused by UV radiation. This supports the suggestion that other molecules besides chlorophyll sensitize or shield algae in the UV portion of the spectrum. This work also suggests that the biological weighting for longterm effects of UV on phytoplankton will be heavily weighted in the UV-B with some weighting in the UV-A region of the spectrum. which implies an $\varepsilon(\lambda)$ that lies somewhere between the examples given in Fig. 1 .

Recently Buhlmann of al. (1987) studied the influence of UV on the ${ }^{1+} \mathrm{C}$-assimilation of phytoplankton. These workers conclude that photoinhibition is caused by $\mathrm{LV}-\mathrm{A}$ radiation and that the additional influence of UV-B is of minor importance. In agreement with Jokiel and York (1984), they find that high intensities of PAR (up to $1800 \mu \mathrm{E} \mathrm{m}^{-2} \mathrm{~s}^{-1}$ ) cause only minor photoinhibition in the absence of UV-A.

Effects on phyloplankton, other than those influencing growth rates, have been observed. Dohler (1985) has shown direct influence of UV-B on protein content. dry weight. and pigment concentration. This work demonstrated that UV-B resulted in chloroplast damage and may also directly affect proteins of the photosynthetic apparatus. For motile microorganisms. Haeder (1987) has shown that both the motility and phototactic orientation can be impaired by UV radiation. For corals. Jokiel ( 1980 ) has shown
Acute exposure to

$\mathrm{UV}$ - $\mathrm{B}$ radiation

significantly depressed

the radiocarbon

estimate of primary

productivity. 
The extreme change

in ozone... produces

a sharp gradient which

creates a UV-B front

analogous to an

oceanographic front. that the current level of ultraviolet radiation directly influences physiology and growth.

\section{Antarctic Ozone Hole and UV-B Fronts}

The recent dramatic reduction in stratospheric ozone over the Antarctic (Stolarski et al., 1986: Schoeberl and Krueger. 1986: Farman et al.. 1985: Connor et al.. 1987: Frederick and Snell. 1988: Lubin et al. . 1989) includes $30-50 \%$ springtime loss resulting in at least a several-fold increase in UV-B incident on the Southern Ocean, compared to preozone hole levels. This has raised concern that the ozone hole may cause increases of ultraviolet radiation at the ocean 's surface and cause harm to aquatic organisms (Voytek, 1989). The debate over the possible consequences of the ozone hole on aquatic life in the Antarctic (Roberts, 1989) illustrates the difficulty in extrapolating laboratory and shipboard findings to predictions of possible impacts at the community and ecosystem level. The extreme change in ozone associated with the hole produces a sharp gradient which creates a UV-B front analogous to an oceanographic front. Comparative studies of UV-B effects across this front may provide a natural laboratory for direct observation of the impact of UV-B on natural phytoplankton communities.

Solar radiation in polar regions is distinguished by low sun elevations and persistent cloud cover. leading to generally lower UV and visible radiation. At the same time. the ratio of diffuse (sky) to global (sky plus direct sun) radiation is high in polar regions, leading to higher reflectances from the ocean surface and slight spectral shifts in the in-water light field. Thus. one might suppose that phytoplankton in Antarctic waters receive absolute levels of UV-B exposure significantly less than levels currently experienced by phytoplankton at lower latitudes. However, a critical issue with respect to light-limited Antarctic phytoplankton is the ratio of biologically weighted fluence rate (UV-B dose) to the photoreactivating $\mathrm{UV}-\mathrm{A}$ and photosynthetically available radiation ( $P A R$ ). These ratios provide a measure of the energy available for UV-B damage to that available for photorepair and/or photosynthesis. The ratio of UV-B to longer wavelengths increases as ozone is depleted in spite of the generally lower incident solar radiation in Antarctic regions. As discussed above, the biological impact of these changing ratios are critically dependent on a choice of the biological weighting function $\varepsilon(\lambda)$ for the photoeffect under consideration.

In order to illustrate the magnitude of spectral irradiance changes caused by the Antarctic ozone hole and to compare these changes with those at lower latitudes, an atmospheric model (Tanre et al., 1979) as well as data from polar latitudes are used to estimate the ratio of UV to PAR without consideration of $\varepsilon(\lambda)$. Fig. 2 shows a plot of the ratio of spectral irradiance to total PAR $\left(\mathrm{E}(\lambda) / \mathrm{E}_{\mathrm{PAR}}\right)$ for the Antarctic. normalized by this same ratio for equatorial latitudes with an ozone concentration of $263 \mathrm{DL}$. A ratio of 1.0 in Fig. 2 indicates that the spectral balance of solar

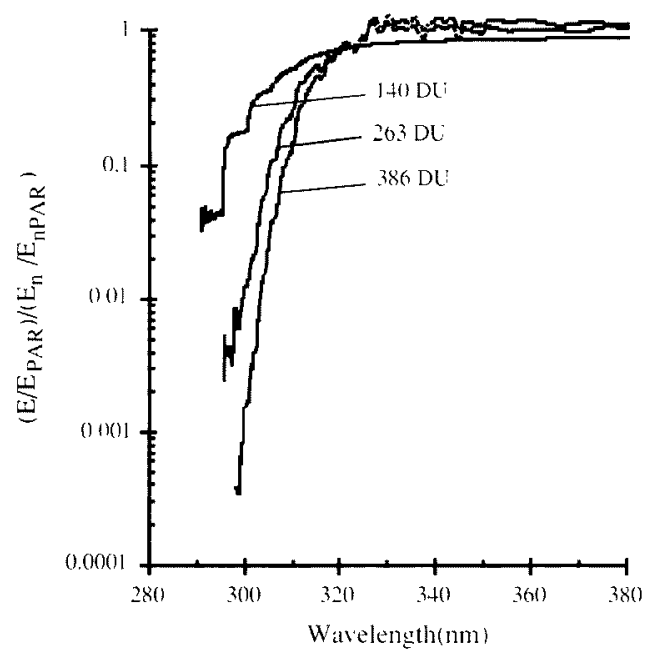

Fig. 2: $E(\lambda) / E_{P, 4 R}$ for the Antarctic (McMurdo Station. solar noon sun angle $\left.71^{\circ}\right)$ divided by $E_{n}(\lambda) / E_{n+k}$ for modeled equatorial latitudes (solar noon sun angle $23^{\circ}$, for the wavelength region $280-380 \mathrm{~nm}$ with ozone concentration of $263 \mathrm{DL}$. Bottom curve for Julian day 29+ (1988) with osone concentration of $380 \mathrm{DU}$ (outside the ozone hole): middle curve for Julian day 281 (1988) with ozone concentration of $263 \mathrm{DU}$ (inside the hole). Data are from NSF-DPP Ut-monitoring program, C.R. Booth, personal communication. Top curve is for a simulated ozone concentration of $1+0 \mathrm{DU}$, near the lowest azone concentration yet observed in the Antartic. using the same atmospheric model used for normalization to equatorial conditions.

radiation passing through the Antarctic atmosphere is comparable to that for low latitudes. A ratio less than 1.0 indicates that the portion of spectral UV in the Antarctic is lower than for equatorial latitudes. We normalize to equatorial latitudes because tropical seas normally experienced the lowest ozone concentrations and, thus, higher ratios of incoming UVB:UVA:PAR than any other ocean areas. The Antarctic data from the National Science Foundation Division of Polar Programs (NSF-DPP) UV monitoring program (C.R. Booth, private communication) corresponds to two ozone regimes: a day "outside" the ozone hole, with a stratospheric ozone concentration of $386 \mathrm{DU}$ (jd 294. 1988) and a day "inside" the ozone hole, with a stratospheric ozone concentration of 263 DU (jd 281. 1988). We simulate a day "inside" the ozone hole, with a stratospheric ozone concentration of $140 \mathrm{DU}$ (near the lowest ozone hole concentrations yet observed), by use of 
the same atmospheric model used to estimate the spectral balance for the equatorial normalization.

A comparison of the two lower curves ( $\mathrm{jd} 281$ and jd 294) shows that the ratio of spectral irradiance inside to outside the hole doubles at $308 \mathrm{~nm}$ and increases thirty-fold at $300 \mathrm{~nm}$. The upper curve. corresponding to an ozone hole concentration near the lowest value yet recorded, indicates that UVB to PAR ratios can approach those of equatorial latitudes despite clouds and low solar elevations. The middle curve (jd 281) shows the correspondence of the $E(\lambda) /$ $E_{P, R}$ ratio for polar to equatorial regions for the same ozone concentrations, but the data from the polar region were for cloudy skies and for low solar elevation (solar zenith angle of $71^{\circ}$ ). These data indicate that the relatively longer atmospheric path lengths for incident radiation in polar regions reduce the UVB to PAR ratio, but not as much as might be expected considering only absolute irradiance levels.

Tilzer and co-workers ( 1985 ) have shown that the photosynthetic capacity (photosynthesis per chl-a at optimum light) and maximum quantum yield of photosynthesis (moles carbon dioxide assimilated per mole light quanta absorbed) are lower, on average, in Antarctic phytoplank ton than for phytoplankton in temperate regions. They suggest that this diminished low-light photosynthesis may be caused by temperature-controlled rate-limiting processes that become dominant in otherwise light-limited situations. If this diminished photosynthetic capacity for Antarctic phytoplankton is true in general. then it is likely that these organisms have less reserve resistance for adaptation and repair of increased UV-B damage. This follows the argument of Calkins and Thordardottir ( 1980 ) that while organisms may have photoadaptive capacity with respect to UV-B, an adaptation to enhanced solar UV reduces the resources available for other purposes. Thus, while the $E(\lambda) / E_{P, R}$ ratio incident on organisms in polar regions may not exceed values already experienced by those in equatorial regions, the current values within the ozone hole are one to two orders of magnitude greater than pre-ozone hole values.

Some of this proportional increase in UV-B will penetrate to ecologically significant depths in Antarctic waters. For example. Smith and Baker (1979) find that for moderately productive waters (chl-a concentration $0.5 \mathrm{mg} \mathrm{m}^{-3}$ ) the effective attenuation coefficient weighted for DNA photodamage is $\mathrm{K}_{\mathrm{DM}} \sim 0.4 \mathrm{~m}^{-1}$. Thus, this mutagenic radiation would penetrate to over $5 \mathrm{~m}$ before being reduced by a factor of ten. Gieshes et $41 .(1989)$ report that $10 \%$ of the UVR $(340 \mathrm{~nm})$ measured above the surface penetrated to $25 \mathrm{~m}$ in Antarctic waters, and Karentz (personnel communication) reports similar findings.

The spatial characteristics of the ozone hole may also influence the potential impact on aquatic systems. Satellite data show substantial motion of the south polar vortex position relative to geographic locations on the surface (see Fig. 3. p. 5). In addition to low ozone concentrations in the south polar vortex ozone hole during austral spring. there is a continuous change in the day-to-day size, shape and position of the vortex (Hofmann et al., 1986). The Nimbus 7 Total Ozone Mapping Spectrometer (TOMS) data (Fig. 3) show relative ozone concentrations with respect to the underlying Antarctic continent for four days in October 1988 (including jd 281 and jd 294) and show how quickly ozone levels can change over any given surface location. The difference in total ozone between the two days at a given geographic location can be nearly a factor of two (i.e.. the difference between being outside or inside the ozone hole). Thus, from a biological perspective, the pattern of surface UV-B exposure at a given location in the Southern Ocean is highly variable with large and rapid changes. It should be emphasized that UV-A and PAR will not change correspondingly in passing from inside to outside the ozone hole. Thus, there is a biological dose gradient corresponding to the ozone gradient (i.e., a UV-B or biological dose front). This front. analogous to oceanographic fronts, provides a unique opportunity to carry out experiments comparing the influence of a change in UV-B stress on natural populations on either side of the front. In particular, the time scales of photosynthetic physiology and photodamage/photorepair processes are such that if the passage of a UV-B front is ecologically significant it would be followed first by changes in the rapid processes (hours) and then by those on longer time scales (days). If such a comparison could separate natural variation from that caused by passage of the LV-B front, it could provide a natural environmental test of the hypothesis that phytoplankton populations in Antarctic waters are endangered by ozone depletion.

\section{Summary}

There is ample laboratory evidence that enhanced UV-B radiation is detrimental to marine organisms and grounds for supposing that enhanced levels of UV-B maty he detrimental to various natural communities of marine organisms. However, the ability to accurately and unambiguously predict effects remains elusive. Estimated impacts range from the insignificant to the catastrophic. Difficulties in quantitatively extrapolating laboratory and shipboard findings to the potential effects of increased UV-B radiation on natural communities include: the natural variability of in-water radiation levels influenced by both physical (e.g. cloud cover and sun elevation) and biological (e.g. in-water biological constituents leading to changes in UV-B penetration) processes; the wide range of aquatic organisms and a natural variability in their development stages, pigmentabsorptive characteristics and sensitivities to UV-B radiation: the possibility that natural communities of algae might quickly adapt to changes in UV flux simply through selection of resistant types; the possibility that light-limited phytoplankton may have photosynthetic light utilization efficiencies that are insufficient to drive the added synthetic reactions
Some of this

proportional increase

in UV-B will penetrate

to ecologically

significant depths

in Antarctic waters. 
required to overcome additional UV-induced damage: the potential for significant indirect effects such as changes in community structure: and the large statistical variance associated with the reproductive success of many aquatic organisms. The uniqueness of the sharp ozone hole UV-B front in the Antarctic. along with knowledge that low-light. low-temperature adapted phytoplankton may be especially vulnerable to enhanced UV-B, may provide the kind of opportunity needed for investigating phytoplankton communities in the field with and without ozone induced UV-B stress. The need is great for direct measures of ultraviolet radiation effects on natural communities in the world's oceans.

\section{Acknowledgements}

This work was supported by NSF Grant DPP 88 17643 and NASA Grant NAGW-290-10. A. Krueger (NASA/GSFC) provided the TOMS data. Pete Peterson contributed analysis of TOMS data. Barbara Prezelin, Sue Weiler, Ron Zaneveld and an unidentified reviewer provided valuable comments.

\section{References}

Brasseur. G. 1987: The endangered orone layer. Emiromment. 29. 6-45.

Buhlmann. B. P. Bassard and U. Uehlinger, 1987: The influence of longwave ultraviolet radiation $(L, V-A)$ on the photosynthetcactivity ${ }^{14} \mathrm{C}$-assimulation) of phytoplank ton. J. 1) Plankton Res. 9/5) 9.35-94.3.

Caldwell, M.M. L.B. Camp, C.W. Warner and S.D. Flint. 1986: Action spectra and their key role in assessing biological consequences of solar UV-B radiation change. In:

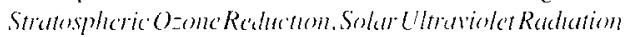
and Plant Life. R.C. Worrest and M.M. Caldwell. eds. Spronger-Verlag. Berlin. 87-111.

Calkins.J. and T. Thordardoltir. 1980: The ecological significance of solar UV radiation on aquatic organisms. Naturc. 283. $563-566$.

Connor. B.J., J.W. Barrett, A. Parrish. P.M. Solomon. R.L. DeZalra. and M. Jaramillo. 1987: Ozone over McMurdo Station, Antarctica, Austral Spring 1986: Altitude profiles for the middle and upper stratospherc. J. Geophys. Res. 92. 13221-13230

Dohler. G.. 1985: Effect of UV-B ridtation (191)-320)m on the nitrogen netabolism of several marine diatoms. I. Plamt Phisol. I/8.391-400.

Farman.J.C.. B.G.Gardiner. and J.D. Shankin, 1985: Large losses of total ozone in Antarctica reveal seasonil ClO/NO interaction, Noture, 315, 207-210.

Frederick. J.E. and FI.E. Snell. 1988: Lltraviolet rudiation levels during the Antarctic spring. Sctonce. 2+1, 438-4+0.

Giese. A.C. 1976: Living with our sun's utrandelet rax. Plenum Press. New York. $185 \mathrm{pp}$.

Gieskes. W.W., R. Heunel, G. Kralay, M.M.Til/er, 1989: The underwater light climate. In: Repents on Polar Researdh. The Expedition Antarkts 1 II// and 2 IEPOS II of Rit Polurstern in /988//989.62. I. Hempel.ed. Alfred Wegener Institute for Polar and Marine Research. 75-\$3.

Haeder. D.P., 1987: Photosensory behavior in procaryotes. Microbiolegical Reviens. 5/. 1-21.

Harris. G.P.. 1978: Photonynthesis, productivity and growth: the physiological ecology of phytoplanktors, Arche tiit Hidrolmolesicie 10.1-171.

Hofmann. D.J.. J.M. Rosen. J.A. Harder and S.R. Roll. 1986: Ozone and aerosol measurements in the springtime Antirutic stratosphere in 1985. Geophlys. Re's. Letters, I.? $1252-1255$.

Jagger. J. 1985: Solar-Ll actions on linme cells. Praeger. New York.
Jerlov, N.G.. 1950: Ultraviolet radiation in the sea. Nature. 100. 111.

Jokiel. P.L. and R.H. York, 1984: Importance of ultravolet radiation in photoinhibition of microal gal growth. Limnol Occencigr..29.192-199.

Jokicl. P.L. 1980: Solar ultraviolet radation and coral reef epilitunal. Science, 207, 11169-1071.

Lenoble, J.. 1956: Etude de la penetration de l'ultraviolet dans lia mer. Ann. Geophlys. 12.16-21.

Lubin. D. J.E. Frederick and A.J. Krueger. 1989: The ultraviolet radiation environment of Antarctica: McMurdo station during September-October 1987.J. Geophys. Res. 94. (in press).

Mees. C.E.K. 1954: The thasy of phomeraphe processes. Mac Nillan Company, New York, 591 pp.

NAS. National Academy of Science. Nattonal Rexearch Council. 1984: Couses andeffects of statusphericuane redution. update 1983. Committee on causes and eftects of changes in stratospheric orone. National Academy Press. Washington. D.C.. $254 \mathrm{pp}$.

NOAA. Nattonal Oceanic and Almospheric Admmistration, 1987 Stratospherie osone: The state of the sciente and NOAA's crarent and future researth. National Oceanic and Atmospheric Administration. Washington. D.C.. $197 \mathrm{pp}$.

Roberts, L... 1489: Does the Ozone Hole Threaten Antaretic Life? Sitence $24+288-289$

Rundel. R.D., 1983: Action spectra and estimation of biologically effective UV radiation. Phwsiol. Plant.. 5\%. 360-360

Schoeberl. M.R. and A.J.Krueger, 1986: Overvew of the Antarcic orone depleton issue. Geophys. Res. Lett. I.3/suppl.) $1191-1192$

Smith. R.C. and K.S. Baher. 1974: Pentration of LV-B and biologically effective done-rates in natural waters.

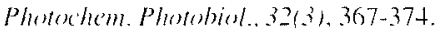

Smith. R.C. and K.S. Baker. 1980: Stratospheric orone, middle ultraviolet radiatton and Carbon-14 measurements of

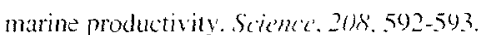

Smith. R.C. and K.S. Baker, 1982: Assesment of the Influence of Enhanced UV-B on Marine Primary Productivity. In: The Rolecy Solar Lltraniolet in Marine Ecosystems.J. Calloms. ed. Plenum Press, New York. 509-53.

Smith. R.C., K.S. Baker. O. Holm-Hannen, and R. Olson, 1980: Pholdinhibition of Photorynthesis and Middle Ultraviolet Radiation in Natural Waters. Phosechem Phomeliol..3/(o). $585-59$ ?

Stolarski, R., A. Krueger. M. Schoeberl. R. McPeters, P. Newman, and J. Alpert. 1986: Vimbus-7 SBUV/TOMS meisurements of the Spring time Antarctic ozone hole Naturc.32: $808-811$.

Tanre. D., M. Herman. P.Y. Deschamps and A. Deleffe. 1979: Atmospheric Modeling for Space Measurements of Ground Reflectunces. Including Bidirectional Properties. Appl Opt. 18. 3587-3596.

Tilzer. M.M. B. Von Bodungen. and V. Smetaceh. 1985: LightDependence of Phytoplankton Photosynthesis in the Antarctic Ocean: Implications for Regulating Productivity: In: Anturetic Nutrient Cocles and Fond Wels. W.R. Sieglried, P.R. Condy, and R.M. Laws, eds. New York. Springer-Verlag. 6(0)68

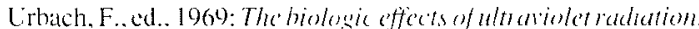
Pergamon Press. London. $7(1) 4 \mathrm{pp}$.

Voytek. M.A.. 1989: Ominous fiture under the ozome hole Assessing bologeral impacts in tharctica. Environmental Detense Fund. $69 \mathrm{pp}$.

Watson. R. 1988: O-one Trends Pancl. Executive Summary. NASA. Washington. D.C.

Worrest. R.C. 1486: The effect of solar LV-B radiation on aquatic systems: An overvicw. In: Elforts of Changes in Stratespheric O-me and Glohul Climate. Orervern. J.G. Titus. ed. US Environmental Protection Agency and United Nutions Environmental Program 1. 175-191.J 